\title{
Special issue on wind turbines and wave energy devices
}

\section{Introduction}

With increasing concerns about climate change and the depletion of fossil fuels, renewable energy has become a topical area for research. To date, significant penetration has taken place of, for example, wind and solar electricity generation systems in an effort to supplement thermal power and other traditional modes of electricity generation. However, the costs associated with these developing technologies are high and favourable feed-in tariffs are usually employed to encourage development. In addition, the variability of the production from such intermittent renewables incurs extra grid integration costs. Nevertheless, wind and other less mature renewable technologies such as wave energy, are required to satisfy increasing world energy demand in a climate neutral and cost effective way. There is therefore an imperative to reduce the cost of wind and wave renewable energy, in order to compete with, and eventually displace, significant amounts of conventional electricity generation plant.

With this economic goal in mind, it is important that wind and wave energy converters incorporate sufficient intelligence to allow them to convert the available energy as efficiently as possible for a given capital investment, while prolonging the life of the wind and wave systems and their components. It is clear that control systems technology has a strong role to play in optimizing the operation of both wind (Johnson, Pao, Balas, \& Fingersh, 2006) and wave (Ringwood, Bacelli, \& Fusco, 2014) conversions systems. This special issue presents a selection of control system approaches and methodologies in both wind and wave application areas as a sample of the spectrum of possibilities. In addition, since both application areas, and the application of control technology in the application areas, have grown up relatively independently, it gives an opportunity to examine the commonality, and contrasts, of both the control challenge of each application area and the nature of the control systems being developed in each domain. In addition, there is an opportunity for cross-pollination between domains, especially in view of the fact that wind energy is now relatively mature, while wave energy is in its infancy.

The issue contains a total of eight papers, spanning the application areas of wind and wave energy.

The opening paper, by Ringwood and Simani, attempts to provide an introduction to both wind and wave energy conversion systems, presenting an overview of the conversion systems and principles, the mathematical models that are used to describe them and the wind and wave resources that drive them, and some control possibilities.
A feature of this paper is the comparisons and contrasts made between the two application areas.

The four papers on wind energy cover particular approaches to the modeling and control of wind turbines. The paper entitled "Gear-box fault detection using time-frequency based methods" by Odgaard and Stoustrup proposes two different time-frequency approaches for monitoring and fault detection in wind turbine gear-boxes using filter-based and Karhunen-Loeve basis schemes. The suggested solution uses measurements already available in wind turbines, thus avoiding the introduction of expensive auxiliary condition monitoring systems. The paper by Fernandez-Canti, Blesa, Tornil-Sina and Puig addresses the problem of fault detection and isolation of wind turbines using a mixed Bayesian/Set membership approach. The main advantage with respect to the classical (Bayesian) approach is that only models of fault-free behavior are exploited. The paper by Badihi, Zhang and Hong presents two active power control schemes that are developed based on adaptive pole placement control and fuzzy gainscheduled proportional-integral control approaches. These strategies provide more efficient regulation of grid frequency and quicker reaction and recovery against frequency events with respect to the active power control schemes available in the literature. The paper entitled "Fault-Tolerant Control of Wind Turbines with Hydrostatic Transmission using Takagi-Sugeno and Sliding Mode Techniques" by Schulte and Gauterin presents an observer design for actuator fault diagnosis and fault-tolerant control scheme of wind turbines with hydrostatic transmission. It is shown that the suggested solution relying on a single observer is able to diagnose, estimate and compensate multiple actuator faults occurring in the wind turbine drive train.

The three papers on wave energy cover control approaches ranging from the more traditional complex-conjugate control to LQG techniques typically found in feedback regulatory system and pseudospectral methods, also appearing in the solution of standard optimal feedback control. The paper entitled "Preliminary consideration of energy storage requirements for sub-optimal reactive control of axisymmetric wave energy devices", by Korde, shows the impedance-matching (reactive control) approach to maximizing energy capture for a wave energy device, but also focusses on the important issue of power smoothing of the raw converted wave power. The paper by Scruggs and Nie adopts an LQG-like approach, where the wave excitation is modeled as a stochastic disturbance, of which only the spectral properties are known. Finally, the paper by Bacelli, Genest and Ringwood shows how a flap-type converter, which exhibits hydrodynamic nonlinearity, can be controlled by a 
pseudospectral approach. A key consideration is the calculation of an optimal control, which takes account of non-ideal efficiency on the mechanical to electrical conversion interface.

In summary, the selection of papers in this special issue, including an overview study, show the relative extent to which control technology has penetrated wind energy, compared to wave. It could reasonably be argued that the greater advancement of control technology in wind energy reflects the greater commercial development of wind energy. This lag in commercial development may be partly explained by the greater technical challenge of efficiently converting the reciprocating energy flux of waves, while the greater inaccessibility of wave energy systems also presents economic challenges. Nevertheless, control problems faced in wave energy are not especially different from those in wind energy, and much can be learned from the more advanced state-of-the-art in wind energy control. Aside from maximizing converted power in view of the physical constraints of the system and a need to maximize the lifetime of device components, there is clear value in fault-tolerant control strategies which respect the relatively inaccessibility of wave energy systems.

\section{References}

Johnson, K. E., Pao, L. Y., Balas, M. J., \& Fingersh, L. J. (2006). Control of variable-speed wind turbines: standard and adaptive techniques for maximizing energy capture. IEEE Control Systems Magazine, 26(3), 70-81.

Ringwood, J. V., Bacelli, G., \& Fusco, F. (2014). Energy-maximizing control of waveenergy converters: The development of control system technology to optimize their operation. IEEE Control Systems Magazine, 34(5), 30-55.

John V. Ringwood

Centre for Ocean Energy Research, Maynooth University, County

Kildare, Ireland

Silvio Simani*

Department of Engineering, University of Ferrara, Ferrara, Italy

* Corresponding author.

E-mail addresses: john.ringwood@eeng.nuim.ie (J.V. Ringwood), silvio.simani@unife.it (S. Simani)

Received 13 September 2015 